\title{
Estudio descriptivo del prejuicio hacia inmigrantes en estudiantes universitarios de CABA y GBA, Córdoba, Salta y Neuquén (AR)
}

\author{
Descriptive approach of prejudice towards immigrants with university \\ students of CABA and GBA, Córdoba, Salta and Neuquén (AR)
}

\author{
Luciana Civalero $^{1}$, Daniela Alonso ${ }^{2}$, Silvina Brussino ${ }^{3}$
}

\begin{abstract}
RESUMEN
Este artículo presenta un abordaje descriptivo sobre el prejuicio hacia inmigrantes en 4 regiones de Argentina. Así, analiza la representación de estudiantes sobre la procedencia de la inmigración en su ciudad, los niveles de prejuicio y sus diferencias en por región y tipo de carrera. También, aborda la relación entre nivel de prejuicio y los años de educación superior y la frecuencia y tipo de contacto con inmigrantes. Para su desarrollo se relevaron muestras no probabilísticas de estudiantes terciarios y universitarios de instituciones públicas y privadas en CABA y GBA, Córdoba, Salta y Neuquén. Los resultados evidencian una percepción cercana a la realidad regional sobre la procedencia de la inmigración, mayores niveles de prejuicio sutil que manifiesto y significativamente más altos en Salta y Neuquén. Además, estudiar carreras sociales y humanas, tener más años de estudios superiores y mayores contactos positivos con inmigrantes se asoció a una reducción de los niveles de prejuicio.
\end{abstract}

Palabras clave: Prejuicio, Inmigración, Educación, Cultura política.

\begin{abstract}
This article presents a descriptive approach on the prejudice towards immigrants in 4 regions of Argentina. Thus, it analyzes the students' representation on the origin of immigration in their city, their prejudice levels and its differences between regions and type of career. It also addresses the relationship between the prejudice level and the amount of years of higher education and the frequency and type of contact with immigrants. For its development, non-probabilistic samples of tertiary and university students from public and private institutions were surveyed in CABA and GBA, Córdoba, Salta and Neuquén. The results show a perception close to the regional reality about the origin of immigration, higher levels of subtle prejudice than blatant prejudice, which was significantly higher in Salta and Neuquén. In addition, studying social and humanistic careers, having more years of higher education and more positive contacts with immigrants was associated with a reduction in the prejudice levels.
\end{abstract}

Keywords: Prejudice, Immigration, Education, Political culture.
Recibido: 29-07-2018 Aceptado: 08-11-2018
Citar: Civalero, L., Alonso, D., \& Brussino, S. (2018). Estudio descriptivo del prejuicio hacia inmigrantes en estudiantes universitarios de CABA y GBA, córdoba, Salta y Neuquén (AR). Investigaciones en Psicología, 23(2), pp. 9-18. doi:10.32824/investigpsicol.a23n2a1

1Universidad Nacional de Córdoba. Facultad de Psicología. Instituto de Investigaciones Psicológicas (IIPsi). Córdoba, Argentina. luciana.civalero@hotmail.com

${ }^{2}$ CONICET - Universidad Nacional de Córdoba. Facultad de Psicología. Instituto de Investigaciones Psicológicas (IIPsi). Córdoba, Argentina. alonsodaniela.psi@gmail.com

${ }^{3}$ CONICET - Universidad Nacional de Córdoba. Facultad de Psicología. Instituto de Investigaciones Psicológicas (IIPsi). Córdoba, Argentina. sbrussino@conicet.gov.ar 


\section{INTRODUCCIÓN}

La inmigración es un fenómeno económica, política y socialmente relevante en el contexto mundial actual. Cada año, millones de personas migran escapando de desastres naturales, persecuciones políticas y guerras o en busca de mejores condiciones económicas o culturales (Brown \& Zagefka, 2011) y, cada vez más, muchísimas de ellas siguen luchando por la conquista de derechos humanos básicos. Si bien en algunos contextos se ha avanzado hacia el reconocimiento de derechos a personas inmigrantes, éstas aún sufren distintos niveles de rechazo por miembros de las mayorías oriundas, especialmente cuando su apariencia física o su forma de vestir revelan su procedencia (Licata, Sanchez-Mazas \& Green, 2011). En este marco, y desde la psicología política en particular, se han analizado dimensiones psicosociales vinculadas a los procesos de integración entre grupos culturalmente diversos (Kessler et al., 2010), principalmente en términos del análisis del prejuicio y los comportamientos discriminatorios.

Desde una conceptualización básica, el prejuicio es definido como "una actitud negativa hacia un grupo socialmente definido y hacia cualquier persona percibida como miembro de ese grupo" (Ashmore, 1970, citado en McLaren, 2003, p. 253). Así, desde su origen involucra procesos intergrupales y de categorización social e implica orientaciones evaluativas cognitivas, afectivas y conductuales (Duckitt, 2003). Estos procesos tienen a acentuar las diferencias entre el grupo de pertenencia y el que se percibe como exogrupo basándose en estereotipos que, a la vez que simplifican la interpretación del mundo social, impactan en actitudes y comportamientos y se ubican en la base del desarrollo de las identidades grupales (Tajfel \& Turner, 1979). Además de hallarse en la base de procesos de categorización social, una connotación negativa de la minoría puede aplicarse como argumento que legitima prácticas discriminatorias.

Respecto del análisis psicosocial de los tipos de prejuicio, la literatura ha identificado que los procesos de democratización y la promoción de valores de tolerancia y no discriminación han impactado en sus formas de expresión (Cárdenas, Gómez, Méndez \& Yáñez, 2011). En este marco, la manifestación del prejuicio habría ido mudado desde expresiones manifiestas de rechazo -muchas veces socialmente censuradas- a otras formas más sutiles y más difíciles de detectar que suelen sostenerse más en la acentuación de las diferencias culturales (Pettigrew \& Meertens, 1995). Sobre las dimensiones sutiles del prejuicio existe cierto cuestionamiento acerca de si efectivamente son una manifestación del mismo. Al respecto, la evidencia empírica sostiene que no sólo se relacionan con las dimensiones explícitas del prejuicio, sino que también predicen actitudes negativas y comportamientos discriminatorios (Pettigrew, 2008; Pettigrew \& Meertens, 2001). En esta línea, es preciso aplicar una definición más amplia del prejuicio que exceda a las actitudes directamente negativas para incluir todas aquellas cuya función sea la de mantener diferencias de estatus y poder entre grupos (Dovidio, Hewstone, Glick, \& Esses, 2010).

Desde este enfoque, proponemos un análisis descriptivo con población estudiantil de Argentina que nos permita caracterizar el prejuicio hacia inmigrantes. Con mayor desarrollo en el contexto regional (i.e. Cárdenas, 2006; Cárdenas et al., 2011; Murillo Muñoz \& Molero Alonso, 2012; Segura-Robles, Gallardo Vigil \& Alemani Arrebola, 2017), su abordaje desde la psicología en Argentina registra menos antecedentes, casi exclusivamente centrados en la religión metropolitana (Ungaretti, 2017; Ungaretti, Etchezahar \& Brussino, 2015). Sin embargo, es uno de los países con mayor porcentaje de población inmigrante en la región y aún la etnia y la nacionalidad se constituyen entre las principales causas de discriminación (Atencio, 2016).

La inmigración ha sido una constante en Argentina, identificándose dos corrientes centrales. En un primer momento, hacia fines del siglo XIX y la primera mitad del siglo XX, Argentina recibe inmigración principalmente de países europeos en crisis, en el marco de una política demográfica planificada por el estado para contribuir al desarrollo económico del país (Benencia, 2004). Así, se percibía como una inmigración deseada y estas personas inmigrantes pudieron incorporarse a procesos de movilidad social ascendente. Posteriormente, principalmente desde la década del '60, Argentina recibe a inmigrantes de países limítrofes quienes adquirieron la connotación de inmigración no deseada, no sólo por provenir de economías en crisis (Benencia, 2004; Pizarro, 2012), sino principalmente porque se los identifica como culturalmente contrastantes con el estereotipo sobrevalorado del ciudadano europeo. Así, se registra la llegada de personas de Bolivia, Chile, Paraguay y, en menor medida, de Uruguay y Brasil, atraídas por ocupaciones temporarias de las distintas regiones. A mediados de la década del 90' se incrementó el flujo migratorio peruano, con perfil migratorio semejante al de países limítrofes (Bologna \& Falcón Aybar, 2012). Así, según el censo de 2010, la procedencia de la población nacida en el extranjero proviene principalmente de cuatro países: Paraguay (30.5\%), Bolivia (19.1\%), Chile (10.6\%) y Perú (8.7\%); que acumulan un $68.9 \%$ del total de los no nativos. Con respecto a los llegados en el último período inter-censal (2001-2010), dentro de la proporción de inmigrantes en relación al total de la población, se destacan quienes provienen de Perú (50\%), Paraguay (38.2\%) y Bolivia (36.7\%), los cuales registraron importantes crecimientos intercensales. (INDEC, 2010).

En este marco, la percepción diferencial de las corrientes migratorias según su origen tiene fuertes bases psicosociales, desde aquellas vinculadas con la situación económica del país, al desarrollo de estereotipos negativos, frecuentemente sostenidos por la representación mediática de estos grupos y por los propios gobiernos como mecanismos de legitimación de políticas públicas discriminatorias y represivas (Cerutti \& Viard, 2012, Pizarro, 2012). Así, es preciso entender que el prejuicio y su expresión en conductas discriminatorias no son 
fenómenos individuales, sino que se originan en el marco de procesos de interacción donde un grupo mayoritario no reconoce a otro grupo, minoritario, como merecedor de estima social (Licata, et al., 2011)

En función de esta perspectiva, nos interesa realizar un abordaje que atienda a la importancia del contexto socio-cultural en el que los grupos se insertan; siendo relevante incluir población estudiantil de la región donde residen la mayor parte de los inmigrantes en Argentina (CABA y GBA), pero también de otras regiones estratégicas que reciben un flujo importante de inmigrantes con trayectorias diferentes: Neuquén, Salta y Córdoba. Las ciudades de Neuquén y Salta han recibido históricamente flujos migratorios principalmente de Chile y Bolivia respectivamente, siendo Neuquén una de las ciudades con mayor proporción de inmigrantes en relación a su población total (7.59\%). Por su parte, como señalábamos, la Ciudad Autónoma de Buenos Aires es la jurisdicción con mayor porcentaje de población nacida en el extranjero en relación a su población total (13.2\%), contando, a su vez, con el $21.1 \%$ del total de del país. Incluimos también al Gran Buenos Aires por la movilidad diaria entre los 24 partidos y la capital y por poseer éste una alta proporción de inmigrantes (7.5\%), albergando al $41.1 \%$ del total de inmigrantes que viven en el país. La Ciudad de Córdoba, por su parte, tuvo un acelerado crecimiento de la población de inmigrantes en los últimos años, atribuido a las redes migratorias que permitieron la circulación de información y facilidades para el establecimiento de familiares y amigos de quienes arribaron con anterioridad (Falcón Aybar \& Bologna, 2013; Magliano, Perissinotti \& Zenklusen, 2013; Pascucci, 2010; Pizarro; 2012). Así, en términos comparativos, Salta y Córdoba presentan una similitud en la proporción de inmigrantes en relación al total de la población; pero la mayor proporción de ellos en Salta llegó antes de 1991 (el 67.3\%), mientras que en Córdoba el $42.8 \%$ de sus inmigrantes llegaron entre 2002 y 2010. Este no es un dato secundario cuando se pretende investigar las concepciones que los habitantes de estas ciudades tienen respecto al establecimiento de extranjeros.

En línea con un análisis comprehensivo de la relevancia del contexto socio-cultural, señalamos también la importancia de analizar y comparar estudiantes de distintas disciplinas y en estadios diferentes de la carrera. Alguna literatura señala que las carreras correspondientes a disciplinas sociales y humanas tendrían una orientación ideológicamente más liberal que aquellas asociadas a las ciencias exactas. En esta línea, Hooge (2017) encontró en una muestra de estudiantes belgas que quienes cursaban carreras de psicología y ciencia sociales tenían actitudes más favorables hacia los inmigrantes que quienes cursaban derecho y economía. Sin embargo, esta diferencia no se incrementaba con el avance de la carrera, por lo cual podría responder más a un proceso de auto-selección previa que a un efecto de socialización de la educación universitaria. Así, es posible que la experiencia educativa en sí misma tenga un efecto en las actitudes intergrupales, independientemente de la carrera. Al respecto, Easter- brook, Kuuppens y Manstead (2016) presentan evidencia de un análisis de series de datos, señalando que la educación formal tiene un efecto positivo en la reducción de actitudes negativas hacia inmigrantes. Sin embargo, alguna literatura sugiere que la documentada reducción de los niveles de prejuicio en los segmentos de mayor nivel educativo se registraría a través de mediciones explícitas, pero no en medidas implícitas (Kuppens \& Spears, 2014): estos grupos manifestarían un apoyo explícito mayor a dimensiones ideológicas igualitarias, pero todavía tendrían reacciones negativas espontáneas hacia inmigrantes o minorías étnicas. Así, estas diferencias podrían ser indicadores tanto de un menor nivel real de prejuicio en estos grupos como de una mayor deseabilidad social en su expresión. En cualquier caso, resulta pertinente controlar no sólo el tipo de carrera, sino también el año de cursado, aspirando a captar el rol de la experiencia educativa en la evolución de este tipo de actitudes.

Otra variable que nos interesa, en relación con los esfuerzos para la reducción de los niveles de prejuicio, es el rol del contacto con inmigrantes en el desarrollo de estas actitudes. Así, con algunas excepciones (i.e. Dejaeghere, Hooghe \& Claes, 2012), la literatura sugiere que el contacto coopera con la reducción del prejuicio, especialmente si se percibe como positivo y significativo y si se analiza a un nivel macro a partir de la aplicación de políticas públicas orientadas a incrementar los intercambios positivos (Christ et al., 2014). En esta línea Pettigrew y Tropp (2006) presentaron un meta-análisis de 515 estudios en 38 naciones en el que señalan que en el $94 \%$ de ellos se registró una asociación negativa entre el contacto y distintos tipos de prejuicio. Además, en un meta-análisis más reciente analizaron las tres vías principales por la cuales el contacto cooperaría en la reducción del prejuicio: el incremento del conocimiento del exogrupo, la reducción de la ansiedad ante el contacto intergrupal y el incremento de la empatía; encontrando que eran las últimas dos -afectivas- las dimensiones más relevantes (Pettigrew \& Tropp, 2008). Sin embargo, es posible que esta relación entre contacto y prejuicio no sea lineal, en la medida en que intervienen otros aspectos coyunturales y el tipo de contacto que se establezca (Christ et al., 2014; Dhont, Roets \& Van Hiel, 2011).

En función de lo expuesto, nuestro objetivo es conocer cuál es la representación sobre la procedencia de la inmigración y los niveles de actitudes prejuiciosas (sutiles y manifiestas) de estudiantes terciarios y universitarios de 4 regiones de Argentina. Además, comparamos si los niveles de prejuicio varían en función de la región y el tipo de carrera y si existe relación entre estos y la cantidad de años de educación terciaria/universitaria y la frecuencia y tipo de contacto con persona inmigrantes. 


\section{MÉTODO}

\section{Tipo de Estudio}

Para abordar el problema de investigación se ha optado por un diseño empírico cuantitativo de tipo transversal, es decir, realizado en un único momento temporal (Montero \& León, 2007). Además, este estudio tiene un carácter descriptivo dado que analiza cómo se manifiesta un fenómeno -en este caso el prejuicio hacia inmigrantesen función de la especificación de sus propiedades relevantes.

\section{Participantes}

La población en estudio fueron estudiantes terciarios y universitarios de instituciones públicas y privadas de las ciudades de CABA y GBA, Córdoba, Salta y Neuquén. Su selección se realizó por un muestreo de tipo no probabilístico autoelegido (Sterba \& Foster, 2008), ya que fue decisión de los y las estudiantes participar o no en el estudio.

La muestra quedó compuesta por 856 estudiantes (CABA y GBA $N=193$, Córdoba $N=253$, Salta $N=200$ y Neuquén $\mathrm{N}=210$ ). El $65.1 \%$ fueron mujeres y la media de edad fue de 23 años. Además, se procuró que se encontraran representadas carreras de distintas áreas para garantizar mayor variabilidad en los perfiles de estudiantes (ciencias exactas, naturales, biológicas, de la salud, agrarias, ingenierías y de materiales un $47.5 \%$ de los casos y ciencias sociales, humanidades y artes $52.9 \%$ ). Finalmente, según el año de cursada, los y las participantes se distribuyeron del siguiente modo: $38.2 \%$ de primer año, $20.5 \%$ segundo año, $16.5 \%$ de tercer año, $8.4 \%$ de cuarto año y $16.3 \%$ de quinto y sexto año.

\section{Instrumentos de Recolección de datos}

Variables Sociodemográficas y académicas: se generó una pregunta cerrada para evaluar sexo y preguntas abiertas para evaluar edad, año de cursado y carrera. Las respuestas a esta última pregunta fueron recodificadas luego según el criterio propuesto por la clasificación de áreas dispuesta por el Consejo Nacional de Investigaciones Científicas y Técnicas.

Contacto con personas inmigrantes: respecto de esta variable, nos interesaba conocer no sólo si la persona había tenido algún contacto con inmigrantes, sino la frecuencia del mismo. En consecuencia, se aplicó un ítem que evaluaba frecuencia de contacto con inmigrantes en una escala que iba de 1 (Nunca) a 5 (Muy a menudo). Además, para aquellos/as participantes que tenían algún contacto con personas inmigrantes, se incluyen 3 ítems adicionales que indagaban -en una escala de 1 a 5 - en qué medida el mismo había sido placentero, cooperativo o superficial.

Procedencia de los inmigrantes en su ciudad: se incluyó una pregunta abierta que solicitaba a los/as participantes que mencionaran la nacionalidad de los grupos de personas en las que piensa cuando habla o escucha hablar de inmigrantes en su ciudad. Esto nos permitió no sólo aproximarnos a la representación sobre la proceden- cia de la inmigración, sino que también se utilizó como priming cognitivo. Así, previo a la aplicación de la escala de prejuicio hacia inmigrantes, se instruyó a los/as participantes para respondiesen pensando en los grupos que mencionaron.

Prejuicio hacia inmigrantes: se aplicó la escala de Pettigrew y Meertens (1995) en su versión adaptada a la Argentina (Etchezahar, Ungaretti \& Brussino, 2015) que consta de 20 ítems que examinan las dimensiones sutiles y manifiestas del prejuicio en función de 5 dimensiones: amenaza-rechazo ( 6 ítems), relaciones cercanas (4 ítems), valores tradicionales (4 ítems), diferencias culturales ( 4 ítems) y emociones positivas ( 2 ítems). Las opciones de respuesta poseen un formato tipo Likert de 5 puntos. Las primeras dos son dimensiones del prejuicio manifiesto, mientras que las últimas 3 evalúan prejuicio sutil. Así, la dimensión amenaza-rechazo involucra las creencias más racistas, como aquellas basadas en la inferioridad genética del exogrupo, desde las que se explica la posición desfavorable de éste en la sociedad y se niega la existencia de discriminación. El segundo componente del prejuicio manifiesto implica una oposición a intimar contacto o mantener relaciones cercanas con el exogrupo. Por su parte, la primera dimensión del prejuicio sutil implicaría la defensa de valores tradicionales propios del endogrupo como parámetro desde el que se determina cuáles son los comportamientos aceptables y necesarios para tener éxito en la sociedad en cuestión. En contraposición, supone que los miembros del exogrupo actúan de maneras impropias. El segundo componente entrañaría la exageración de las diferencias culturales, siendo éste, y no una inferioridad genética, el motivo que justifica la posición desfavorecida del exogrupo. Finalmente, el tercer componente conlleva la negación encubierta de respuestas emocionales positivas hacia el exogrupo (Pettigrew \& Meertens, 1995).

Para este estudio debió eliminarse un ítem de la dimensión amenaza-rechazo que obtuvo valores de curtosis por encima de 3,5 y un ítem de la dimensión valores tradicionales que perjudicaba su consistencia interna. Así, la escala quedó compuesta por 18 ítems, estructura que fue validada a través de un análisis factorial confirmatorio estimado con el método de máxima verosimilitud $(\mathrm{CFI}=.95 ; \mathrm{GFI}=.94 ; \mathrm{RMSEA}=.053)$ y cuyas dimensiones mostraron niveles de confiabilidad aceptables (Aron \& Aron, 2001): (1) amenaza-rechazo $(\alpha=.75)$, (2) relaciones cercanas $(\alpha=.79)$, (3) valores tradicionales $(\alpha=.61)$ (4) diferencias Culturales $(\alpha=.75)$ y (5) emociones positivas $(\mathrm{r}=.53, \mathrm{p}<.001)$.

\section{Procedimiento}

Para la recolección de los datos se utilizó un cuestionario autoadministrado que fue completado por los y las estudiantes en el espacio de clases con presencia de una entrevistadora capacitada que brindó las indicaciones para realizarlo y estuvo disponible para la resolución de dudas. Previo a su administración se brindó información sobre el estudio y sus objetivos, enfatizando en el carácter voluntario de la participación y garantizando la confiden- 
cialidad y anonimato en el tratamiento de los datos. Asimismo, se señaló que podían abandonar el estudio cuando lo deseasen.

\section{Análisis de Datos}

Los datos fueron procesados utilizando los paquetes estadísticos SPSS 21 y AMOS 19. Se realizaron análisis descriptivos univariados para conocer la distribución de los datos en la muestra. Para describir los niveles de prejuicio se crearon rangos con los puntajes brutos de la sub-escalas de modo que se consideraban altos niveles de prejuicio superiores a dos desviaciones estándar por encima de la media, medios-altos hasta dos desviaciones estándar por encima de la media, medios-bajos hasta dos desviaciones estándar por debajo de la media y bajos más de dos desviaciones estándar por debajo de la media.

Adicionalmente, para comparar niveles de prejuicio en las distintas ciudades, se efectuó un análisis de la varianza de un factor (ANOVA) con grupos de tamaños desiguales utilizando los puntajes brutos de las subescalas. Asimismo, para estimar qué medias difieren significativamente entre sí, se realizaron comparaciones múltiples post-hoc con una probabilidad de error tipo I fijado en < .05 utilizando la prueba de Bonferroni. Para el caso de la comparación de grupos de estudiantes según el tipo de carrera, y teniendo en cuenta que se contaba con dos categorías, se aplicó una prueba t de diferencia de medias para muestras independientes. Además, se estimaron análisis de correlación bivariados utilizando el coeficiente de relación Gamma (apropiado para variables ordinales) para conocer si había relación entre el nivel de prejuicio hacia inmigrantes y el año de cursada de la carrera y la frecuencia y tipo de contacto con inmigrantes.

\section{RESULTADOS}

A partir del ítem que solicitaba que especifiquen en grupos de personas de qué países piensa cuando escucha hablar de los inmigrantes en su ciudad, se calcularon las frecuencias de las menciones a cada grupo computando sólo las primeras cinco respuestas de cada participante. Así, se obtuvieron un total de 2119 menciones. En la tabla 1 exponemos los porcentajes de las menciones sobre el total de respuestas. Para mayor claridad en la lectura de los datos, se recogen en la tabla los países que obtuvieron más de un $5 \%$ de las menciones, agrupando los restantes por regiones.
Tabla 1. Frecuencias y Porcentajes de Menciones de los Grupos de Inmigrantes

\begin{tabular}{lcc}
\hline & F & $\%$ \\
\hline Latinoamérica & 635 & 29.97 \\
$\quad$ Bolivia & 315 & 14.86 \\
Perú & 256 & 12.08 \\
$\quad$ Chile & 251 & 11.84 \\
$\quad$ Paraguay & 110 & 5.19 \\
$\quad$ Colombia & 227 & 10.69 \\
$\quad$ Otros países latinoamericanos & 132 & 6.24 \\
Europa Central & 101 & 4.77 \\
Asia Oriental & 26 & 1.23 \\
Asia Occidental (Medio Oriente) & 37 & 1.75 \\
África & 29 & 1.38 \\
Otros (EE.UU., India, Rusia, Nueva Zelanda) & 2119 & 100 \\
\hline TOTAL DE MENCIONES & & \\
\hline
\end{tabular}

Estos resultados muestran que los países más mencionados en general fueron: Bolivia (30\% del total de menciones), Perú (15\%), Chile (12\%), Paraguay (12\%) y Colombia (5\%). De este modo, el $84 \%$ de las respuestas correspondieron a países latinoamericanos, $6 \%$ a países europeos (principalmente España, Italia, Alemania y Francia) y $6 \%$ a países de Asia (principalmente China, Siria, Corea y Japón).

Adicionalmente -teniendo en cuenta que cada participante podía reportar hasta cinco grupos inmigrantes- nos interesaba conocer qué porcentaje de los y las participantes mencionaba a cada grupo. En la tabla 2 se exponen estos porcentajes correspondientes a los 4 grupos más mencionados tanto sobre el total de casos como sobre el total de cada una de las sub-muestras.

Tabla 2. Porcentaje de Participantes que nombraron los 4 grupos de inmigrantes con más menciones

\begin{tabular}{lccccc}
\hline & $\begin{array}{r}\text { Muestra } \\
\text { General }\end{array}$ & Córdoba & CABA-GBA & Neuquén & Salta \\
\hline Bolivia & 74.8 & 71.2 & 72.5 & 73.3 & 83.5 \\
Perú & 37.6 & 59.3 & 55.5 & 11.4 & 20.5 \\
Paraguay & 31 & 20.2 & 55.4 & 29.5 & 25 \\
Chile & 31.3 & 15.6 & 15.6 & 70.4 & 26 \\
\hline
\end{tabular}

Para el total de la muestra (856 casos), el 74.8\% de los y las participantes mencionó a Bolivia cómo país de procedencia de las personas en las que piensa al considerar el tema de la inmigración, un 37.6\% refirió a Perú, un $31.4 \%$ a Paraguay y un $31.3 \%$ a Chile. Además, en todas las sub-muestras Bolivia fue el país con mayor porcentaje de menciones (entre un $71.2 \%$ y $83.5 \%$ ). Sin embargo, 
en las de Córdoba y Buenos Aires el segundo grupo más mencionado fue Perú (59.3\% y $55.5 \%$ respectivamente), mientras que en Salta y Neuquén fue Chile $(26 \%$ y $70.4 \%$ respectivamente). Efectivamente, esto coincide con que Neuquén es una de las ciudades que recibe efectivamente mayor inmigración de procedencia chilena. Asimismo, Chile está entre los países menos mencionados en las ciudades de Córdoba y Buenos Aires (15.5\% y 15.6\% respectivamente). Finalmente, se destaca que el grupo Paraguay tiene un alto porcentaje de menciones en Buenos Aires (55.4\%).
Niveles de prejuicio hacia inmigrantes y diferencias entre las ciudades en estudio

Para poder establecer la presencia de respuestas prejuiciosas, a partir de las puntuaciones de los participantes en cada una de las dimensiones de esta variable, se crearon rangos que reflejaran distintos niveles de prejuicio. En la tabla 3 se muestran los resultados correspondientes a los niveles bajos, medio-bajos, medio-altos y altos de prejuicio en las 5 sub-dimensiones de la variable; sin embargo, para nuestra descripción de los resultados nos centraremos en los segmentos medio-altos (hasta dos desviaciones estándar por encima de la media), y altos (valores que superaran las dos desviaciones estándar).

Tabla 3. Puntuaciones Medias, Desviación Estándar y Niveles de Prejuicio hacia inmigrantes.

\begin{tabular}{lcccccc}
\hline & M & DS & \multicolumn{3}{c}{$\%$} \\
\cline { 5 - 6 } & & & Bajo & Medio-Bajo & Medio-Alto & Alto \\
\hline Prejuicio Manifiesto & 10.74 & 4.39 & 0 & 60 & 37.8 & 2.3 \\
Amenaza-rechazo & 4.7 & 2.75 & 0 & 74.6 & 19.3 & 6.2 \\
Relaciones cercanas & & & & & & \\
Prejuicio Sutil & 8.25 & 2.9 & 0 & 53.1 & 45.8 & 1.2 \\
Valores tradicionales & 11.7 & 3.5 & 12.8 & 53.6 & 29.3 & 4.4 \\
Diferencias culturales & 5.15 & 1.9 & 5.8 & 49.8 & 44.3 & 0 \\
Emociones positivas & & & & & & 0 \\
\hline
\end{tabular}

Para la muestra total, la dimensión que recogió mayores porcentajes de prejuicio fue valores tradicionales -prejuicio sutil- con un $47 \%$ de las respuestas en rangos considerados medio-altos y altos de prejuicio. En segundo lugar, emociones positivas contó con un $44 \%$ de respuestas en los rangos medio-altos, sin registrarse casos en el rango alto. Hasta aquí, destacamos que son las dimensiones sutiles del prejuicio las que recogen porcentajes más elevados en los rangos altos. Por último, como dato saliente, la dimensión amenaza-rechazo -prejuicio manifiesto- se ubica en tercer lugar y concentra un $40.1 \%$ de respuestas entre niveles medio-altos y alto de prejuicio.

Por otra parte, para dar cuenta de las posibles diferencias en estos niveles de prejuicio entre ciudades (en este caso utilizando el puntaje directo) se realizó un análisis de varianza para grupos de tamaños desiguales que arrojó diferencias significativas en todas las dimensiones, siendo las muestras de las ciudades de Salta y Neuquén las que evidenciaron mayores niveles de prejuicio (tabla 4).

Tabla 4. Puntuaciones Medias y Significación de las diferencias en los niveles de Prejuicio según la ciudad de residencia.

\begin{tabular}{|c|c|c|c|c|c|c|c|c|c|}
\hline & \multicolumn{2}{|c|}{ Córdoba } & \multicolumn{2}{|c|}{ CABA y GBA } & \multicolumn{2}{|c|}{ Neuquén } & \multicolumn{2}{|c|}{ Salta } & \multirow[t]{2}{*}{$\mathrm{F}$} \\
\hline & M & SD & M & SD & M & SD & M & SD & \\
\hline \multicolumn{10}{|l|}{ Prejuicio Manifiesto } \\
\hline Amenaza-rechazo & 8.49 & 3.30 & 10.39 & 4.54 & 11.32 & 4.16 & 13.31 & 4.18 & $55.20^{* * *}$ \\
\hline Relaciones cercanas & 3.84 & 1.78 & 4.74 & 2.92 & 4.78 & 2.82 & 5.68 & 3.15 & $17.65^{* * *}$ \\
\hline \multicolumn{10}{|l|}{ Prejuicio Sutil } \\
\hline Valores tradicionales & 7.42 & 2.74 & 7.91 & 3.18 & 8.46 & 2.73 & 9.41 & 2.63 & $19.95^{* * *}$ \\
\hline Diferencias culturales & 11.18 & 3.4 & 11.19 & 3.82 & 11.79 & 3.23 & 12.79 & 3.27 & $10.11^{* * *}$ \\
\hline Emociones positivas & 4.58 & 1.63 & 5.50 & 2.20 & 5.24 & 1.79 & 5.41 & 1.85 & $11.73^{* * *}$ \\
\hline
\end{tabular}


Asimismo, a través de un análisis post-hoc utilizando la prueba de Bonferroni, se describe cuáles fueron los grupos que tuvieron diferencias significativas entre sí. Así, las diferencias fueron significativas para las dimensiones del prejuicio manifiesto entre todas las sub-muestras $(p \leq .000)$, salvo entre las ciudades de Neuquén y CABAGBA que no resultaron significativas. Por su parte, las dimensiones del prejuicio sutil (sub-escalas valores tradicionales y diferencias culturales) reportaron diferencias significativas entre la sub-muestra de Salta y el resto de las sub-muestras ( $p \leq .000$, salvo en el caso de la diferencia con Neuquén $p \leq .05$ ). Cabe señalar que en la dimensión emociones positivas, sólo fue significativa la diferen- cia de Córdoba en comparación al resto de las submuestras $(p \leq .000)$.

Diferencias en los niveles de prejuicio en función del tipo de carrera y relación con el año de cursado y la frecuencia del contacto con inmigrantes

En la tabla 5 se exponen las puntuaciones medias, desviación estándar y resultado de la prueba t en función del tipo de carrera. Las diferencias son significativas para todas las dimensiones del prejuicio, siendo sus niveles más elevados para la muestra de estudiantes de ciencias exactas y naturales en todos los casos.

Tabla 5. Puntuación Media (M), desviación estándar (SD) y prueba de diferencia de medias $(t)$ en niveles de prejuicio según el tipo de carrera.

\begin{tabular}{|c|c|c|c|c|c|}
\hline & \multicolumn{2}{|c|}{ Exactas y Naturales } & \multicolumn{2}{|c|}{ Humanas y Sociales } & \multirow[b]{2}{*}{$\mathrm{t}$} \\
\hline & M & SD & M & SD & \\
\hline \multicolumn{6}{|l|}{ Prejuicio Manifiesto } \\
\hline Amenaza-Rechazo & 11.87 & 4.34 & 9.74 & 4.19 & $7.32^{* * *}$ \\
\hline Relaciones Cercanas & 5.01 & 2.94 & 4.43 & 2.54 & $3.08^{* *}$ \\
\hline \multicolumn{6}{|l|}{ Prejuicio Sutil } \\
\hline Valores Tradicionales & 8.80 & 2.83 & 7.76 & 2.89 & $5.29^{* * *}$ \\
\hline Diferencias Culturales & 12.21 & 3.51 & 11.29 & 3.40 & $3.88^{* * *}$ \\
\hline Emociones Positivas & 5.43 & 1.95 & 4.89 & 1.81 & $4.15^{* * *}$ \\
\hline $\mathbf{N}$ & 404 & & & 455 & \\
\hline
\end{tabular}

Finalmente, analizamos el rol del año de cursada y la frecuencia y tipo de contacto en relación con el prejuicio hacia inmigrantes. Es preciso mencionar que el $17 \%$ de los/as participantes no había tenido contacto con inmigrantes en ninguna ocasión y el $30.3 \%$ tenía poco contacto. Como contracara, un $25 \%$ tenía un contacto muy frecuente con personas inmigrantes. De cualquier modo, entre los que sí habían tenido algún contacto $(\mathrm{N}=711)$, más del $50 \%$ lo identificaba como algo o muy placentero y algo o muy cooperativo y sólo el $5.7 \%$ lo señalaba como algo o muy superficial. En la tabla 6 se muestran los coeficientes de correlación entre el prejuicio hacia inmigrantes y año de cursada y frecuencia del contacto. Respecto de las relaciones estimadas, estas fueron negativas y moderadas pero significativas. Así, en todos los casos, una mayor cantidad de años de estudios superiores y mayor frecuencia del contacto con inmigrantes se asociaron con una disminución del prejuicio, tanto en sus dimensiones sutiles como, principalmente, manifiestas. Además, cuando se estimaron las correlaciones según el tipo de contacto, encontramos que el contacto placentero y cooperativo se relacionaba significativamente con menores niveles de prejuicio en todas las dimensiones, mientras que el contacto superficial se asociaba con mayor prejuicio. 
Tabla 6. Relación entre las dimensiones del prejuicio hacia inmigrantes y la frecuencia del Contacto y Año de Cursada

\begin{tabular}{|c|c|c|c|c|c|}
\hline & $\begin{array}{l}\text { Año de Cursada } \\
\qquad(\mathrm{N}=856)\end{array}$ & $\begin{array}{c}\text { Frecuencia del } \\
\text { Contacto } \\
(\mathrm{N}=856)\end{array}$ & $\begin{array}{c}\text { Contacto } \\
\text { Placentero } \\
(\mathrm{N}=711)\end{array}$ & $\begin{array}{c}\text { Contacto } \\
\text { Cooperativo } \\
(\mathrm{N}=711)\end{array}$ & $\begin{array}{c}\text { Contacto } \\
\text { Superficial } \\
(\mathrm{N}=711)\end{array}$ \\
\hline \multicolumn{6}{|l|}{ Prejuicio Manifiesto } \\
\hline Amenaza-Rechazo & $-.31^{* * *}$ & $-.19^{* * *}$ & $-.34^{* * *}$ & $-.25^{* * *}$ & $.28^{* * *}$ \\
\hline Relaciones Cercanas & $-.20^{* * *}$ & $-.21^{* * *}$ & $-.37^{* * *}$ & $-.31^{* * *}$ & $.22^{* * *}$ \\
\hline \multicolumn{6}{|l|}{ Prejuicio Sutil } \\
\hline Valores Tradicionales & $-.21^{* * *}$ & $-.11^{* * *}$ & $-.25^{* * *}$ & $-.18^{* * *}$ & $.19^{* * *}$ \\
\hline Diferencias Culturales & $-.10^{* *}$ & $-.07^{*}$ & $-.23^{* * *}$ & $-.16^{* * *}$ & $.17^{* * *}$ \\
\hline Emociones Positivas & $-.11^{* * *}$ & $-.19^{* * *}$ & $-.37^{* * *}$ & $-.35^{* * *}$ & $.24^{* * *}$ \\
\hline
\end{tabular}

${ }^{* * *} p \leq .001 ;{ }^{* *} p \leq .01$

\section{DISCUSIÓN}

El objetivo de este trabajo, de carácter descriptivo, fue conocer los niveles de prejuicio hacia inmigrantes entre estudiantes terciarios y universitarios, analizando las dinámicas que adquieren en distintas regiones de Argentina, así como el posible rol de la educación universitaria y los contactos directos con personas inmigrantes en la reducción de estas actitudes negativas. Si bien no analizamos relaciones de tipo explicativas, sí pudimos aportar información relevante que reconoce la importancia del contexto respecto de actitudes intergrupales como el prejuicio.

En primer lugar, nuestros datos permitieron corroborar que, en general, la representación de los y las estudiantes sobre la nacionalidad de procedencia de los inmigrantes en su ciudad coincide con los grupos de mayor afluencia según los datos censales (INDEC, 2010). Así, por ejemplo, el grupo chilenos recogía más de un $70 \%$ de menciones en la muestra neuquina, siendo menos prominente en las restantes. Por su parte, si bien el grupo bolivianos era el más mencionado en todas las muestras, recogía aún más menciones en la muestra salteña; al tiempo que el grupo paraguayos tenía más menciones en CABA y GBA, donde se encuentra más representado entre la población migrante. Esto nos permite concluir que los y las estudiantes tienen una representación relativamente ajustada a la realidad de su contexto.

Por otra parte, el abordaje de los niveles de prejuicio dio cuenta de la prominencia de las dimensiones sutiles del prejuicio por sobre los aspectos manifiestos. Esto coincide con la literatura previa que sostiene que en el mundo occidental las actitudes prejuiciosas de carácter más sutil (también denominadas como racismo moderno o prejuicio simbólico) tienen una mayor prevalencia que las de carácter manifiesto y que ello se debe, al menos en parte, a que las formas de evaluación del prejuicio sutil suelen presentarse como menos ofensivas y, con ello, generan menos reactividad (Pedersen \& Walker, 1997). En este sentido, la dimensión que manifestó menos respuestas prejuiciosas fue justamente aquella que refería a la disposición a mantener contacto con personas inmigrantes. Sin embargo, es preciso destacar que -en las dimensiones sutiles de prejuicio y en una dimensión de prejuicio manifiesto en la muestra salteña- las puntuaciones medias se ubicaron por encima de la media teórica, alertándonos sobre la persistencia de niveles de moderados a altos de prejuicio en esta población. Esto es particularmente relevante atendiendo a que se trata de una población de alto nivel educativo -que la literatura sugiere que expresa menores niveles de prejuicio que la población general (Kuppens \& Spears, 2014; Meeusen, de Vroome \& Hooghe, 2013) -, tratándose además de futuros/as profesionales, muchos y muchas de las cuales podrán luego tener responsabilidades vinculadas a la educación, la salud y el abordaje de la inclusión y la desigualdad y sus consecuencias.

También, este trabajo nos permitió aportar variabilidad regional al abordaje del prejuicio al incluir muestras de 4 ciudades de argentina con trayectorias sociales, económicas y de inmigración diferentes. Este tipo de abordajes se anclan en una perspectiva de cultura política que reconozca la heterogeneidad hacia el interior del país que frecuentemente resulta invisibilizada en tanto los estudios nacionales tienden a favorecer una sobre-representación de ciudadanos y ciudadanas localizados en Buenos Aires (Cao, Favaro \& Iuorno, 2011). Así, nuestros resultados mostraron diferencias significativas en los niveles de prejuicio en distintas regiones, siendo más elevados en las ciudades de Salta y Neuquén que en CABA y GBA y, principalmente, en Córdoba. Además, estas diferencias eran mayores en una de las dimensiones manifiestas de prejuicio hacia inmigrantes (amenazarechazo) que recogió niveles particularmente altos. Es preciso mencionar que estas ciudades son las que limitan geográficamente con los países que los y las estudiantes más mencionaron como origen de los inmigrantes (Bolivia y Chile respectivamente). Además, registran flujos de migración de larga data y sostenidos en el tiempo. Asimis- 
mo, la muestra de Córdoba fue la que manifestó inferiores niveles de prejuicio en todas las dimensiones estudiadas, siendo además la que tiene un flujo de inmigración más diverso y que se ha intensificado con los años (INDEC, 2010). En línea con estos resultados, es relevante indagar qué aspectos de la cultura política se asocian con estos mayores niveles de prejuicio y analizar cómo intervenir sobre esta problemática.

Finalmente, nuestro estudio permitió realizar una primera aproximación al rol de la experiencia educativa y el contacto con personas inmigrantes en la manifestación del prejuicio. Así -de modo consistente con la literatura antecedente- se encontró que mayor cantidad de años de educación universitaria se asociaban con una disminución de los niveles de prejuicio hacia inmigrantes, principalmente en sus dimensiones manifiestas (Easterbrook et al., 2016). Esto es consistente con lo que proponen Kuppens y Spears (2014) respecto del rol de la educación en la disminución de la expresión manifiesta del prejuicio a partir de la adhesión a valores igualitarios; que convive con una persistencia de reacciones negativas menos directas hacia inmigrantes o minorías étnicas. Por otra parte, también encontramos diferencias en los niveles de prejuicio entre estudiantes de carreras sociales y humanas y estudiantes de carreras exactas, naturales e ingenierías; siendo estos últimos más prejuiciosos/as. Al respecto, no podemos descartar la hipótesis de Hooghe (2017) que señala que estas diferencias pueden deberse más a un proceso previo de autoselección que a la experiencia educativa en sí. De hecho, en nuestra muestra, la experiencia educativa se asocia con menor nivel de prejuicio en todos los casos.

Por su parte, la tesis del contacto (Pettigrew \& Tropp, 2006) parece hallar sustento también en nuestros datos: mientras más contacto los y las estudiantes tenían con personas inmigrantes, menores niveles de prejuicio manifestaban. Además, también en línea con los antecedentes (Christ et al., 2014; Dhont, Roets \& Van Hiel, 2011) encontramos que no todo contacto es igual y que el tipo y calidad son dimensiones relevantes al momento de considerar su efectividad. En este sentido, según esperábamos, el contacto cooperativo y placentero mostraron relaciones negativas y significativas con el nivel de prejuicio hacia inmigrantes; sugiriendo que éste puede cooperar a la reducción de las actitudes negativas hacia esos grupos. Sin embargo, cuando el contacto se percibía como superficial, no sólo no cooperaba en la reducción del prejuicio, sino que se relacionaba positivamente con éste. Este hallazgo es particularmente relevante dado que muchas de las intervenciones orientadas a la inclusión e integración en contextos multiculturales se sostienen en el efecto potencialmente beneficioso del contacto (Lemmer \& Wagner, 2015). En este sentido, es preciso que las experiencias de contacto sean positivas y significativas si se espera que sean potencialmente efectivas en la reducción de actitudes intergrupales negativas.

En función de lo expuesto, entendemos que futuros estudios podrán abordar las dimensiones culturales e ideológicas que permitan explicar las diferencias en los niveles de prejuicio en distintas regiones. Además, es relevante analizar las implicaciones comportamentales diferenciales de las expresiones manifiestas y sutiles del prejuicio; pensando en el desarrollo de abordajes macro y micro sociales para intervenir sobre esta problemática que recrudece en los últimos años.

\section{REFERENCIAS}

Benencia, R. (2004). La existencia de modelos históricos contrapuestos en la integración de los migrantes en la sociedad argentina. Amérique Latine Histoire et Mémoire. Les Cahiers ALHIM, 9. Recuperado de http://journals.openedition.org/alhim/430

Bologna, E., \& Falcón Aybar, M. C. (octubre, 2012). Tendencias de la migración peruana y boliviana en Córdoba. Un análisis a partir de datos censales. Trabajo presentado en el V Congreso de la Asociación Latinoamericana de Población, Montevideo, Uruguay. Recuperado de http://www.alapop.org/Congreso2012/DOCSFINAIS_PDF/ALAP_2012_FINAL451.pdf

Brown, R., \& Zagefka, H. (2011). The Dynamics of Acculturation: An Intergroup Perspective. Advances in Experimental Social Psychology, 44, 129-184.

Cao, H., Favaro, O. \& Luorno, G. (2011). Las provincias argentinas. Estado, territorio y sociedad en las últimas décadas. Neuquén: CEHEPYC, Centro de Estudios Históricos de Estado, Política y Cultura.

Cárdenas, M. (2006). «Y verás cómo quieren en chile...»: Un estudio sobre el prejuicio hacia los inmigrantes bolivianos por parte de los jóvenes chilenos. Ultima década, 14(24), 99-124.

Cárdenas, M., Gómez, F., Méndez, L., \& Yáñez, S. (2011). Reporte de los niveles de prejuicio sutil y manifiesto hacia los inmigrantes bolivianos y análisis de su relación con variables psicosociales. Psicoperspectivas, 10(1), 125-143.

Cerutti, A., \& Viard, G. (2012). De bandera, abanderados y extranjeros en las escuelas neuquinas. Consideraciones sobre el trabamiento del problema en el diario "La Mañana del Sur”. Scripta Ethnologica, 34, 31-38.

Christ, O., Schmid, K., Lolliot, S., Swart, H., Stolle, D., Tausch, N., Ramiah, A. A., Wagner, U., Vertovec, S., \& Hewstone, M. (2014). Contextual effect of positive intergroup contact on outgroup prejudice. Proceedings of the National Academy of Sciences, 111(11), 3996-4000.

Dejaeghere, Y., Hooghe, M., \& Claes, E. (2012). Do ethnically diverse schools reduce ethnocentrism? A two-year panel study among majority group late adolescents in Belgian schools. International Journal of Intercultural Relations, 36(1), 108-117.

Dhont, K., Roets, A., \& Van Hiel, A. (2011). Opening closed minds: The combined effects of intergroup contact and need for closure on prejudice. Personality and Social Psychology $\mathrm{Bu}$ lletin, 37(4), 514-528.

Dovidio, J. F., Hewstone, M., Glick, P., \& Esses, V. M. (2010). Prejudice, stereotyping and discrimination: theoretical and empirical overview. En J. F. Dovidio, M. Hewstone, P. Glick, \& V. M. Esses (Eds.), SAGE handbook of prejudice, stereotyping, and discrimination (pp. 3-28). London: Sage.

Duckitt, J. (2003). Prejudice and intergroup hostility. En D. Sears, L. Huddy, \& R. Jervis (Eds.), Oxford handbook of political psychology (pp. 559 - 600). Oxford: Oxford University Press. 
Easterbrook, M. J., Kuppens, T., \& Manstead, A. S. (2016). The education effect: Higher educational qualifications are robustly associated with beneficial personal and socio-political outcomes. Social Indicators Research, 126(3), 1261-1298.

Etchezahar, E., Ungaretti, J., \& Brussino, S. (2015). Modelo predictivo del prejuicio sutil y manifiesto hacia los inmigrantes bolivianos: agresión autoritaria, dominancia e ideología politica como factores predisponentes. Reporte de investigación inédito.

Falcón Aybar, M. D. C., \& Bologna, E. (2013). Migrantes antiguos y recientes: una perspectiva comparada de la migración peruana a Córdoba, Argentina. Migraciones internacionales, 7(1), 235-266.

Hooghe, M. (2017). Diferencias en las actitudes políticas de estudiantes universitarios de acuerdo a las disciplinas académicas. Resultados de una encuesta aplicada en una universidad belga. Revista de la Educación Superior, 46(181), 1-18.

Atencio, C. (2016). Migrantes y discriminación. Buenos Aires: INADI. Recuperado de http://www.inadi.gob.ar/contenidos-digitales/wp-content/uploads/2016/03/migrantes-y-discriminacion.pdf

Instituto Nacional de Estadística y Censo [INDEC] (2010). Censo Nacional de Población, Hogares y Viviendas 2010. Censo del Bicentenario. Pueblos originarios. Buenos Aires: INDEC. Recuperado de https://www.indec.gob.ar/ftp/cuadros/poblacion/ censo2010_tomo1.pdf

Kessler, T., Mummendey, A., Funke, F., Brown, R., Binder, J., Zagefka, H., Leyens, J. P., Demoulin, S., Maquil, A. (2010). We all live in Germany but... Ingroup projection, group-based emotions and prejudice against immigrants. European Journal of Social Psychology, 40(6), 985-997.

Kuppens, T., \& Spears, R. (2014). You don't have to be well-educated to be an aversive racist, but it helps. Social science research, 45, 211-223.

Lemmer, G., \& Wagner, U. (2015). Can we really reduce ethnic prejudice outside the lab? A meta-analysis of direct and indirect contact interventions. European Journal of Social Psychology, 45(2), 152-168.

Licata, L., Sanchez-Mazas, M., \& Green, E. G. (2011). Identity, immigration, and prejudice in Europe: a recognition approach. En S. J. Schwartz, \& V. L. Vignoles (Eds.), Handbook of Identity Theory and Research (pp. 895-916). New York: Springer.

Magliano, M. J., Perissinotti, M. V., \& Zenklusen, D. (2013). Mujeres bolivianas y peruanas en la migración hacia Argentina: especificidades de las trayectorias laborales en el servicio doméstico remunerado en Córdoba. Anuario Americanista Europeo, 11, 71-91.

Meeusen, C., de Vroome, T., \& Hooghe, M. (2013). How does education have an impact on ethnocentrism? A structural equation analysis of cognitive, occupational status and network mechanisms. International Journal of Intercultural Relations, 37(5), 507-522.
McLaren, L. M. (2003). Anti-Immigrant Prejudice in Europe: Contact, Threat Perception, and Preferences for the Exclusion of Migrants. Social Forces, 81(3), 909-936.

Montero, I., \& León, O. G. (2007). Guía para nombrar los estudios de investigación en Psicología. International Journal of Clinical and Health Psychology, 7(3), 847-862.

Murillo Muñoz, J. \& Molero Alonso, F. (2012). La satisfacción vital: su relación con el prejuicio, la identidad nacional, la autoestima y el bienestar material, en inmigrantes. Acta Colombiana de Psicología, 15(2), 99-108.

Pascucci, S. S. (2010). Migraciones y clase social: Un análisis crítico de la bibliografía sobre inmigrantes bolivianos en Argentina. Miradas en Movimiento, 4, 6-35.

Pedersen, A., \& Walker, I. (1997). Prejudice against Australian Aborigines: Old-fashioned and modern forms. European Journal of Social Psychology, 27(5), 561-587.

Pettigrew, T. F. (2008). Intergroup prejudice: Its causes and cures. Actualidades en Psicología, 22(109), 115-124.

Pettigrew, T. F., \& Meertens, R. W. (1995). Subtle and blatant prejudice in Western Europe. Europeanjournal of social psychology, 25(1), 57-75.

Pettigrew, T. F., \& Meertens, R. W. (2001). In defense of the subtle prejudice concept: A retort. European Journal of Social Psychology, 31(3), 299-309.

Pettigrew, T. F., \& Tropp, L. R. (2006). A meta-analytic test of intergroup contact theory. Journal of personality and social psychology, 90(5), 751.

Pettigrew, T. F., \& Tropp, L. R. (2008). How does intergroup contact reduce prejudice? Meta-analytic tests of three mediators. European Journal of Social Psychology, 38(6), 922-934.

Pizarro, C. A. (2012). El racismo en los discursos de los patrones argentinos sobre inmigrantes laborales bolivianos: Estudio de caso en un lugar de trabajo en Córdoba, Argentina. Convergencia, 19(60), 225-285.

Segura-Robles, A., Gallardo-Vigil, M. Á., \& Alemany-Arrebola, I. (2017). Prejuicio sutil y manifiesto: Un estudio comparado con alumnado del grado de Educación Social de las facultades de Melilla y Huelva. ReiDoCrea, 6, 130-139.

Tajfel, H., \& Turner, J. C. (1979). An integrative theory of intergroup conflict. The social psychology of intergroup relations, 33(47), 74.

Ungaretti, J. (2017). La evaluación del prejuicio sutil y manifiesto hacia colectivos indígenas e inmigrantes de países limítrofes. Calidad de Vida y Salud, 10(1).

Ungaretti, J., Etchezahar, E., \& Brussino, S. (2015). La percepción de peligro y competencia como factores predictores del prejuicio hacia diferentes grupos sociales. Escritos de Psicología (Internet), 8(1), 30-37. 